\title{
Wilms' tumor: experience of regional children's hospital in serbia
}

\begin{abstract}
Wilms' tumor-nephroblastoma is the most common malignant tumor of the kidney in children. It belongs to disontogenetic tumors, developing in early embryogenesis. Typical age for diagnosis is between 1st and 3rd year of life but it can be found until 15th year. Wilms' tumor develops more often in children with other congenital anomalies. It is caused by mutations of Wilms' tumor suppressor gene WT1 (11p13) and WT2 (11p15). Clinically it presents most often with enlargement of abdomen and haematuria. Diagnosis is made by the ultrasonography and computerized tomography. Patients are treated according to tumor histology and stage. Aim of the study was to present the prevalence of Wilms tumor in regional childrens hospital and identify factors correlating to bad prognossis. The study was retrospective and presents the period of 16 years, from 1995 to 2010. It was conducted in the Center for Hematology and Oncology in the Institute for Children and Youth Health Care of Vojvodina-Novi Sad, Serbia. Prevalence of Wilms' tumor was 23 children in 16 years with higher incidence in years: 2003, 2005, 2009 and 2010. Children diagnosed in stage I and II had better 2 year disease free and overall survival than children in stage III and IV. Also when diagnosis was made in less than 1 month, 2 year disease free and overall survival was better than in group where the diagnosis was made in a period after 1 month. In management of Wilms' tumor there is still controversy whether the children should receive upfront chemotherapy versus primarily operation.
\end{abstract}

Keywords: wilms' tumor, nephroblastoma, children
Volume 4 Issue 5 - 2016

\author{
Maja Kovacevic,' Stojsic Mirjana, ${ }^{2}$ \\ Konstantinidis $\mathrm{Nada}^{3}$ \\ 'Department of Nephrology, Institute for Children and Youth \\ Health Care of Vojvodina, Serbia \\ ${ }^{2}$ Department of Hematooncology, Institute for Children and \\ Youth Health Care of Vojvodina, Serbia \\ ${ }^{3}$ Department of Gastroenterohepatology, Institute for Children \\ and Youth Health Care of Vojvodina, Serbia
}

Correspondence: Maja Kovacevic, Paediatric nephrologist, Institute for Children and Youth Health Care of Vojvodina, Novi Sad, Serbia, Email dr.maja.kovacevic@gmail.com

Received: March 08, 2016 | Published: April 15, 2016

\section{Introduction}

Malignancy in children presents the major cause of death due to disease between the ages of 1 and 15 year. Tumors differ from tumors in adult age in their nature, localization and prognosis. The basic challenge of pediatric oncology is adjustment of therapy to the biological nature and stage of malignant process, while not interfering with growth and development of the child. The most frequent tumors in children are acute lymphoblast leukemia, tumors of the central nervous system and sarcomas. Tumors of the kidney present $10 \%$ of tumors in the first 5 years of life and 5\% of tumors between the 5-9 years of age. Pediatric oncology management in Europe is according to the SIOP protocols. SIOP (Société Internationale d'Oncologie Pédiatrique) is international association of pediatric oncologist which was founded in Geneva, Switzerland in 1960. ${ }^{1,2}$

Tumors in children originate from the tissue (e.g. haematopoietic, nervous and connective tissues) that is not in direct contact with environment, which emphasizes importance of host factors. Environmental factors in cancerogenesis are drugs, viruses, obesity and nutrition rich in fats. ${ }^{2-4}$ Ionizing radiation such as $\mathrm{X}$-rays, gamma rays, and particle radiation from radioactive substances can predispose child to cancerogenesis. Ions formed in tissue cells under the influence of such radiation are highly reactive and can rupture DNA strands, causing mutations. ${ }^{5}$

\section{Wilms' tumor}

Wilms' tumor-nephroblastoma is the most common malignant tumor of the kidney in children. It is an embryonic tumor, developing from primitive metanephric blastema. Wilms' tumor belongs to disontogenetic tumors, developing in early embryogenesis which is why in pathohistological examination one can find abortive glomeruls and tubules but also a cartilage, bone and muscle tissue.
Incidence of Wilms' tumor is 8 children per million. Typical age for diagnosis is between $1^{\text {st }}$ and $3^{\text {rd }}$ year of life but it can be found until $15^{\text {th }}$ year. It is the only tumor that can develop bilaterally in $5 \%$ of cases and multifocaly. It develops more often in children with aniridia, congenital malformation of kidney and urogenital tract (CAKUT), mental retardation, hemihypertrophy (Beckwith-Wiedemann syndrome), nephropathy and genital abnormalities (Denys-Drash syndrome) or trisomy of $18^{\text {th }}$ chromosome. $^{6}$

\section{Genetical basis}

Most of the children have mutations of Wilms' tumor suppressor gene WT1 (11p13) and WT2 (11p15). WT1 gene can be found in locus $11 \mathrm{p} 13$. His role is to control transcription of genes in normal development of the kidney. Mutation of WT1 usually happens in early embryogenesis and leads to formation of „nephrogenic rest” which is a cluster of blast and stromal cells with a potential for later malignant alteration. „Nephrogenic rest” can be found only in Wilms tumor and that is important in differential diagnosis compared to other tumors.

WT2 is second important gene. It is localized in locus 11p15 and its role in embryogenesis is still not clearly defined. Some define him as insulin like growth factor. WT2 mutation also leads to BeckwithWiedemann syndrome.

Autosomal dominant inheritance is proven in $15-20 \%$ of children with Wilms' tumor. ${ }^{6}$

\section{Clinical manifestation}

Abdomen enlargement is often the first sign. It can be noticed by the parents. It is palpated as a firm mass which doesn't cross , linea alba" of the abdomen. Tumor can be symptomatic and asymptomatic. Symptoms can be abdominal pain, fever, anemia, haematuria and arterial hypertension (caused by pressure on renal artery leading to 
ischemia and hypersecretion of renin). Sometimes hypertension and haematuria may mislead to diagnose of acute poststreptococcal glomerulonephritis. Tumor can cause acute abdomen if it ruptures into the abdominal cavity. If it spreads to the inferior vena cava or right atrium it can clinically present with heart congestion symptoms: hepatomegaly and ascites. Small percent can present with paraneoplastic syndrome, hemorrhage due to acquired Von Wille brand disease or Cushing syndrome because of high ACTH production. ${ }^{6}$

\section{Diagnosis of the tumor}

Wilms' tumor can be diagnosed by the means of ultrasonography or computerized tomography (CT scan). It is very important to determine the function of the contralateral kidney and in some cases presence of another congenital anomaly besides tumor. With computed tomography, one can determine if the tumor process disseminated into the renal vein, inferior vena cava, right atrium or liver, spleen and rectum. Vascularization of the tumor and lymph nodes enlargement also should be estimated as much as the X-ray of the lung to determine metastatic changes. Magnetic resonance, selective renal arteriography and echocardiography also could be performed.

Opinions about the biopsy of the tumor process still differ. One of the attitudes is that the biopsy can enlarge the staging of the tumor. Some think that it should be done if the clinical presentation of Wilms' tumor is not specific with urinary tract infection or sepsis or if the child with tumor is older than 5 or 6 years. Some of the indications for biopsy of the tumor would be the presence of calcifications in the mass or extrarenal localization. Absolute contraindications for biopsy of the tumor are the child which is younger than 6 months, presence of cysts without renal parenchyma. In those cases operative approach is indicated. ${ }^{7}$

\section{Differential diagnose}

In the differential diagnose it is important to consider neuroblastoma, rhabdomyosarcoma, leiomyosarcoma, renal cell sarcoma, fibrosarcoma, hydronephrosis, polycystic kidney, hemorrhage in suprarenal glands and renal vein thrombosis.

\section{Patohistology of Wilms' tumor and prognosis}

Classical patohistology of Wilms' tumor is from three components with different proportions of blastemic, epithelial and stromal part. Presence of anaplasia in any of three components and most often on blastemic is only criteria for bad prognosis. ${ }^{6}$

Classification of Wilms' tumor in children is based on correlation of histological characteristics and survival. SIOP (Stockholm) Working Classification of Renal Tumors of Childhood (1994) included three groups in relation with prognosis: low risk, intermediate risk and high risk tumors. New SIOP trials still recognizes the three risk groups with different types in each of them, but for treatment only three types of nephroblastoma need to be recognize: completely necrotic (low risk tumor), blastemal (high risk tumor) and others (intermediate risk tumors). Patients are treated according to tumor histology and stage. Biological nature of the tumor determines its sensitivity to chemotherapy and is very important for survival. The results of the latest SIOP trials showed that certain histological features which remain after preoperative chemotherapy, such as blastema are of prognostic significance. ${ }^{6}$ Low risk group tumors can be diagnosed only with radiology and treated surgically. In general prognosis is good. One of those tumors is necrotic nephroblastoma. Intermediate risk tumors are epithelial and stromal. They usually develop in younger children and are low stage in the moment of diagnosis compared with blastemic types. Anaplastic tumors are rare in the first 6 month of life and in general before first year of life. They are more common after 5 years of life.

\section{Staging}

In the management of Wilms' tumor there are two large groups with different opinion: the NWTS (from North America) and the SIOP (Europe). SIOP gives preoperative chemotherapy, removal of tumor and than does staging. The NWTS group initially removes tumor and then stages it and proceeds with chemotherapy and radiation therapy. The staging system is shown in Table $1.8,9$

Table I Staging system for Wilms' tumor

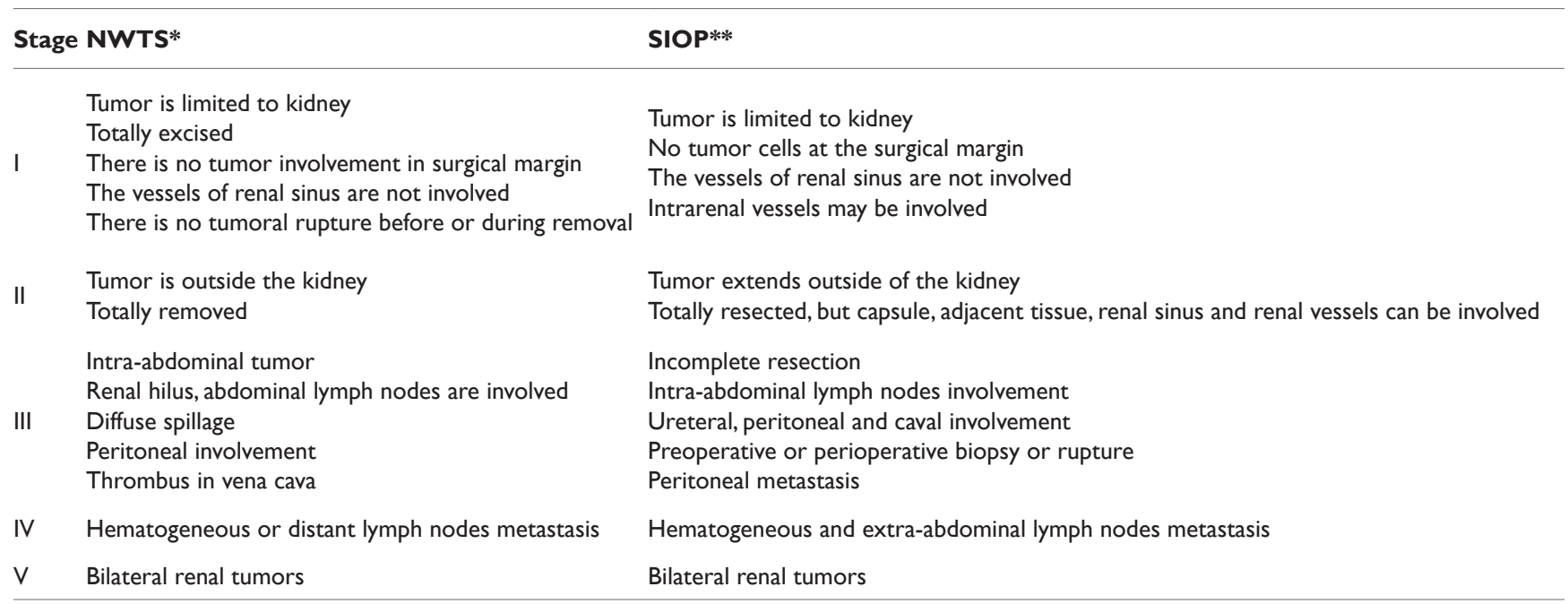

*National Wilms' Tumor Study Group (NWTS), Pediatric Oncology Group in the USA, Canada.

**European society for Pediatric Oncology (SIOP). 


\section{Management of Wilms' tumor}

There is controversy whether the children should receive upfront chemotherapy which is option by SIOP versus primarily operation option by NWTS.

SIOP attitude towards management of Wilms' tumor in children older than 6 months and younger than 18 years is primarily treatment with chemotherapy. The same attitude is advised also in other childhood tumors like neuroblastoma, rhabdomyosarcoma and hepatoblastoma. The aim of preoperative chemotherapy is to reduce size of the tumor and destroy micro metastasis which can't be seen with standard radiology methods. Trials which include preoperative chemotherapy have shown that the presence of necrotic tumor or chemotherapy induced changes in the renal sinus or perirenal fat can be ignored for distinguishing between stage I and II, but if present at resection margins or lymph nodes, it should be regarded as stage III. ${ }^{10}$ In cases where body mass of a child is less than 12 kilograms, dose of chemotherapy is reduced to $2 / 3$ of the dose defined by protocol. Children who are less than 6 months old usually have better prognosis, most of them are in stage I, so they are not always given upfront chemotherapy, but only nephrectomy.

Radiation therapy is indicated for management of high risk tumors and lung metastases. Anaplastic, bilateral or recurrent tumors don't show the same response to treatment protocols. Isolated lung irradiation is advised in children who do not respond to other forms of therapy. Abdominal irradiation is usually poorly tolerated. Hematogeneous metastases of Wilms' tumor occur in lung, liver, bone, brain, or lymph nodes outside the abdomen but lungs are the most common site of metastases.

\section{Objective of the Study}

Objective of the study was to present the prevalence of Wilms' tumor in only children's hospital for the region of Vojvodina in Serbia, with a population of about 2 million people and identify factors correlating to bad prognosis.

\section{Methods}

The study was retrospective and presents the period of 16 years, from 1995 to 2010. It was conducted in the Center for Hematology and Oncology in the Institute for Children and Youth Health Care of Vojvodina-Novi Sad, Serbia. Statistical methodology was almost entirely descriptive due to small sample.

\section{Results}

The study included 23 children. Minimum age was 2 months; maximum age was 84 months ( 7 years). Median age was 37.8 months (3 years and 1 month). In the group of children younger than 5 years, overall survival of 5 years had $81.2 \%$ (13/23) compared with $71.4 \%$ $(5 / 23)$ who died. In the group of children older than 5 years $18.8 \%$ (3/5) survived compared with 28.6\% (2/5) who died (Figure 1).

Prevalence was 23 children in 16 years, with the maximum incidence in 2003. With 5 new cases, in the year 2005. There were 4 new and 3 in 1995, 2009 and 2010 (Figure 2).

$60.9 \%(14 / 23)$ were girls and $39.1 \%(9 / 23)$ were boys. $13 \%(3 / 23)$ had another congenital malformation and $87 \%$ (20/23) didn't.

$26.1 \%(6 / 23)$ were in stage I, $47.8 \%(11 / 23)$ were stage II, $17.4 \%$ $(4 / 23)$ were stage III and $8.7 \%$ (2/23) had stage IV Wilms' tumor. There was no bilateral Wilms tumor, stage V. Overall in $69.6 \%$
$(16 / 23)$ the histological finding was good and in $30.4 \%(7 / 23)$ it was bad (Figure 3).

After they were diagnosed with Wilms tumor, $65.2 \%(15 / 23)$ of children had initial surgery and $34.8 \%(8 / 23)$ had initial chemotherapy. There survival functions: 2 year Disease Free Survival (DFS) and 2 year Overall Survival (OS) are presented graphicly below with Kaplan-eier (Figure 4).

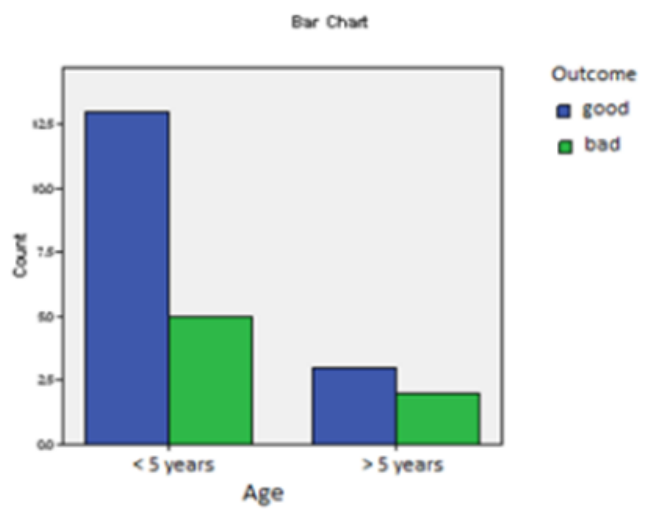

Figure I Statistical Methodology for overall survival of children's.

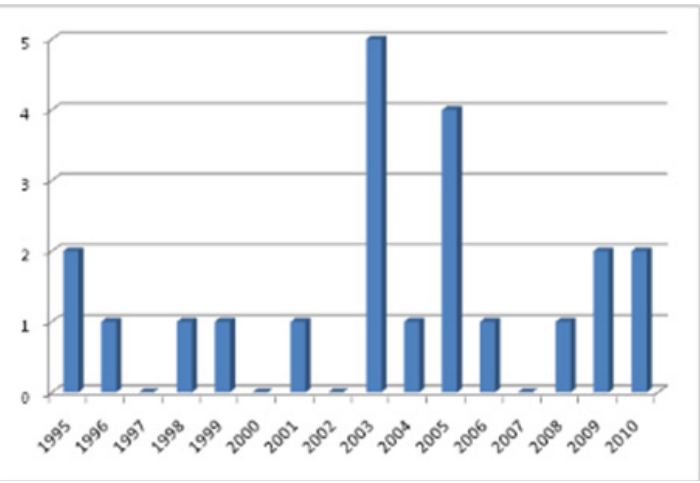

Figure 2 Prevalence was 23 children in 16 years, with the maximum incidence in 2003. With 5 new cases, in the year 2005. There were 4 new and 3 in 1995, 2009 and 2010.

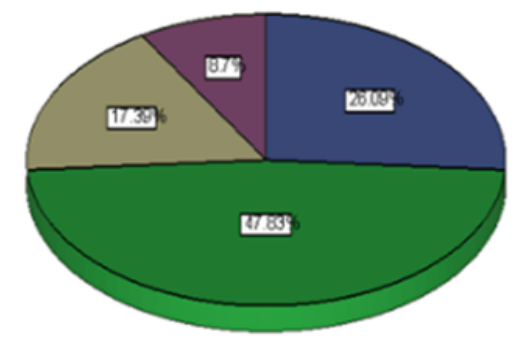

Figure 3 Histological finding of Wilms' tumor. 26.1\% (6/23) were in stage I, 47.8\% (I I/23) were stage II, I7.4\% (4/23) were stage III and $8.7 \%(2 / 23)$ had stage IV Wilms' tumor. There was no bilateral Wilms tumor, stage V. Overall in $69.6 \%(16 / 23)$ the histological finding was good and in $30.4 \%(7 / 23)$ it was bad. 

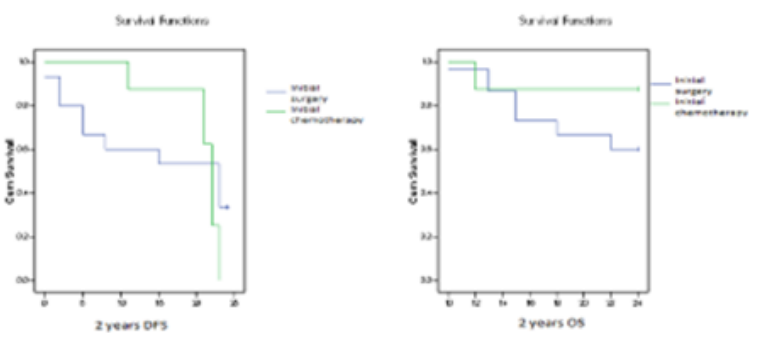

Figure 4 There survival functions: 2 year Disease Free Survival DFS) and 2 year Overall Survival (OS) are presented graphically by using Kaplan-Meier.

2 year Disease Free Survival (DFS) and 2 year Overall Survival (OS) are was also analyzed compared with staging and presented with Kaplan-Meier curve (Figure 5).

Duration of symptoms before diagnosis was compared with 2 year Disease Free Survival and 2 year Overall Survival (OS) and graphically presented with Kaplan-Meier (Figure 6).
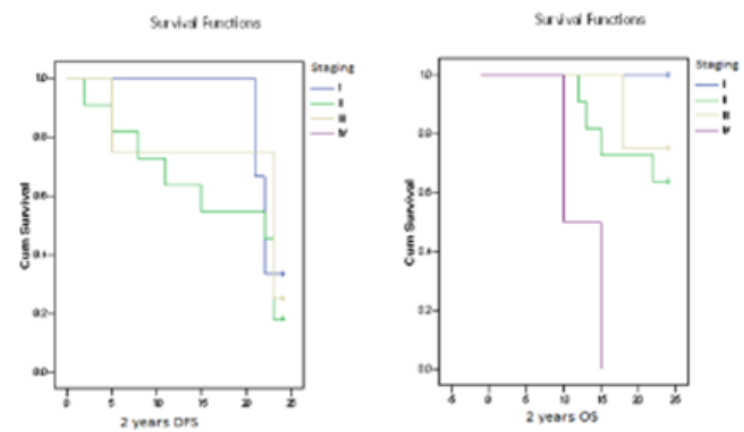

Figure 52 year Disease Free Survival (DFS) and 2 year Overall Survival (OS) are was also analyzed compared with staging and presented with KaplanMeier curve.
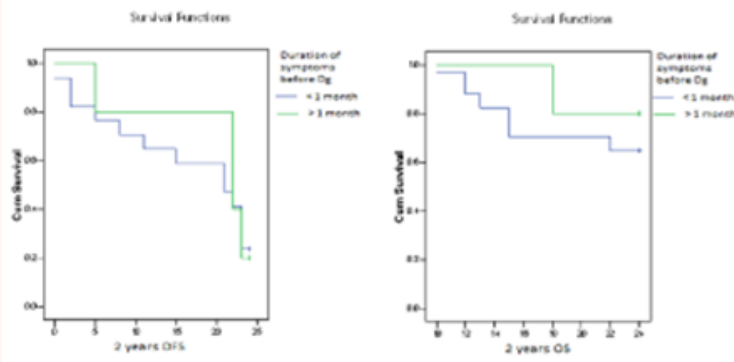

Figure 6 Duration of symptoms before diagnosis was compared with 2 year Disease Free Survival and 2 year Overall Survival (OS) and graphically presented with Kaplan-Meier.

\section{Discussion}

The median age of presentation of Wilms' tumor was 3 years and 1 month. Prevalence was 23 children in 16 years. Incidence was lesser then estimated average of 8 children in 1 year, but growing in last couple of years.

In stage II and I were diagnosed $73.9 \%$ of children. In a group of children were diagnosis of Wilms' tumor was made in less than 1 month, 2 year disease free and overall survival was better than in group where the diagnosis was made in a period after 1 month. Early diagnosis in stage I and within period of one month is crucial for better outcome, so it is important to consider tumor as a possible cause in every child presenting with haematuria.

Better 2 year disease free and overall survival was found in children who were initially treated with chemotherapy compared with initial surgical removal of tumor.

\section{Conclusion}

Wilms' tumor-nephroblastoma is the most common malignant tumor of the kidney in children. Typical age of presentation is between 1 st and 3rd year of life. It is important for every pediatrician to make diagnose of Wilms' tumor in stage I and within the first month of symptoms like enlarged abdomen or haematuria. Beside stage another important factor for prognosis is tumor histology. High risk tumors with bad prognosis are anaplastic. In the management of the Wilms' tumor, children can receive upfront chemotherapy versus primarily operation. In the operative approach it is essential to be done by pediatric urologist so there won't be tumoral rupture before or during removal of the tumor. The Overall 2 and 5-year Survival is estimated to be approximately $90 \%$ for the children diagnosed and treated on time.

\section{Conflicts of Interest}

The authors declare no conflict of interest.

\section{Acknowledgments}

None.

\section{Funding}

None.

\section{References}

1. Crist MW. Neoplastic Diseases and Tumors. In: Behrman ER, et al (Eds.), Nelson Textbook of Pediatrics. $16^{\text {th }}$ edn. WB Saunders Company, Philadelphia, USA. 2000;1531-1542.

2. Zergollern Lj, Onkologija. Zergollern Ljiljana i sur. Pedijatrija, Univerzitet u Zagrebu, Zagreb, Croatia. 1994;1497-1508.

3. Alberts B, Bray D, Lewis J, et al. Basic Genetic Mechanism. Molecular Biology of the Cell. $3^{\mathrm{r}} \mathrm{d}$ edn. Garland Publishing, New York, USA, 1994;223-291.

4. Ford HL, Sclafani RA, Degregori J. Cell cycle regulatory cascades. In: Stein GS \& Pardee AB eds., Cell cycle and growth control: biomolecular regulation and cancer, 2nd edn, Wiley-Liss, Hoboken, USA. 2004;p.4267.

5. Guyton C, Hall J. Textbook of Medical Physiology. 12th edn, Saunders, USA. 2011.

6. Đuričić S. Wilmsov tumor-nastanak. Dijagnostika, lečenje i prognoza, Zadužbina Andrejević, Beograd, Serbia. 2007.

7. Vujanic GM, Kelsey A, Mitchell C, et al. The role of biopsy in the diagnosis of renal tumors of childhood: Results of the UKCCSG Wilms Tumor Study 3. Med Pediatr Oncol. 2003;40(1):18-22.

8. Vujanić GM, Sandstedt B, Harms D, et al. Revised International Society of Paediatric Oncology (SIOP) working classification of renal tumors of childhood. Med Pediatr Oncol. 2002;38(2):79-82.

9. Kalapurakai JA, Dome JS, Perlman EJ, et al. Management of Wilms' tumor: current practice and future goals. Lancet Oncol. 2004;5(1):3746.

10. Mitchell C, Jones PM, Kelsey A, et al. The treatment of Wilms' tumor: results of the United Kingdom Children's cancer study group (UKCCSG). Second Wilms' tumor study. Br J Cancer. 2000;83(5):602608 . 